\title{
Author Correction: Denary oxide nanoparticles as highly stable catalysts for methane combustion
}

Tangyuan Li, Yonggang Yao, Zhennan Huang, Pengfei Xie D, Zhenyu Liu, Menghao Yang, Jinlong Gao (D), Kaizhu Zeng, Alexandra H. Brozena (D), Glenn Pastel, Miaolun Jiao, Qi Dong (D), Jiaqi Dai, Shuke Li, Han Zong, Miaofang Chi (D), Jian Luo, Yifei Mo (D), Guofeng Wang (D), Chao Wang (D), Reza Shahbazian-Yassar (D) and Liangbing Hu (D)

Correction to: Nature Catalysis https://doi.org/10.1038/s41929-020-00554-1, published online 11 January 2021.

In the version of this Article originally published, in Fig. $3 \mathrm{f}$ the bottom-right panel labelled ' $2 \times 10^{6} \mathrm{MC}$ steps' wrongly showed a duplicate of the bottom-left panel labelled ' 0 MC step'. This error has now been corrected and the corrected panel is shown below.
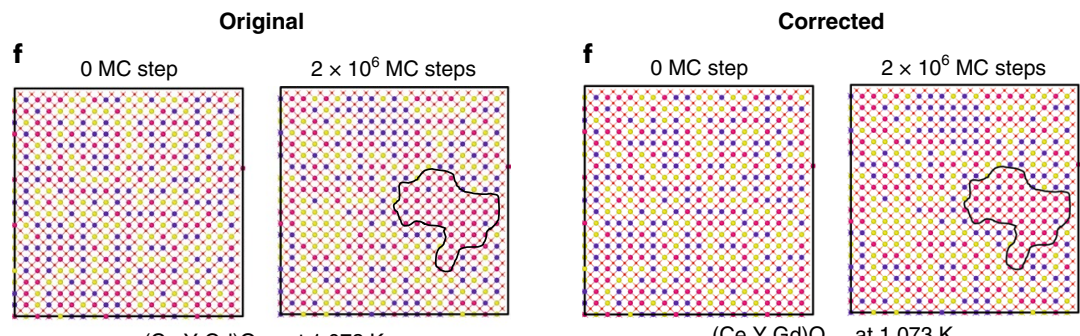

$(\mathrm{Ce}, \mathrm{Y}, \mathrm{Gd}) \mathrm{O}_{2-x}$ at $1,073 \mathrm{~K}$

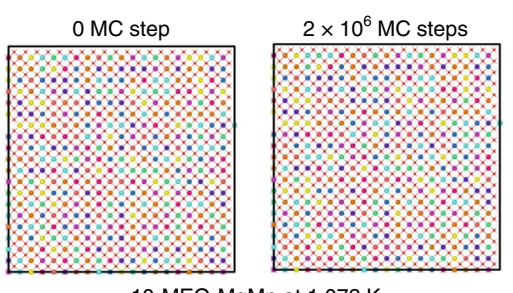

0 MC step $2 \times 10^{6} \mathrm{MC}$ steps
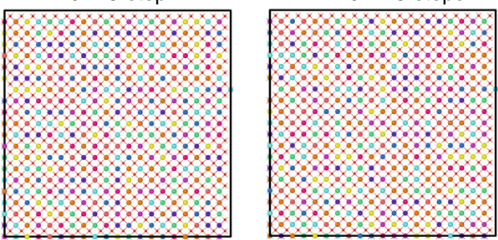

10-MEO-MgMn at 1,073 K

Fig. 3f | Original and corrected.

Published online: 12 April 2021

https://doi.org/10.1038/s41929-021-00613-1

(c) The Author(s), under exclusive licence to Springer Nature Limited 2021 